\title{
INVARIANTS OF BI-LIPSCHITZ EQUIVALENCE OF REAL ANALYTIC FUNCTIONS
}

\author{
JEAN-PIERRE HENRY \\ Centre de Mathématiques (Unité associé au CNRS no. 169) \\ École Polytechnique, F-91128 Palaiseau Cedex, France \\ E-mail: henry@cmat.polytechnique.fr
}

\section{ADAM PARUSIŃSKI}

Département de Mathématiques, U.M.R. 6093 du C.N.R.S, Université d'Angers

2, bd Lavoisier, 49045 Angers Cedex, France

E-mail: parus@tonton.univ-angers.fr

\begin{abstract}
We construct an invariant of the bi-Lipschitz equivalence of analytic function germs $\left(\mathbb{R}^{n}, 0\right) \rightarrow(\mathbb{R}, 0)$ that varies continuously in many analytic families. This shows that the bi-Lipschitz equivalence of analytic function germs admits continuous moduli. For a germ $f$ the invariant is given in terms of the leading coefficients of the asymptotic expansions of $f$ along the sets where the size of $|x||\operatorname{grad} f(x)|$ is comparable to the size of $|f(x)|$.
\end{abstract}

Introduction. Consider a one parameter family of germs $f_{t}(x, y):\left(\mathbb{R}^{2}, 0\right) \rightarrow(\mathbb{R}, 0)$, $t \in \mathbb{R}$, given by

$$
f_{t}(x, y)=f(x, y, t)=x^{3}-3 t x y^{4}+y^{6} .
$$

We shall show that if $t \neq t^{\prime}, t, t^{\prime}>0$, then $f_{t}$ and $f_{t^{\prime}}$ are not bi-Lipschitz equivalent, that is, there is no germ of bi-Lipschitz homeomorphism $h:\left(\mathbb{R}^{2}, 0\right) \rightarrow\left(\mathbb{R}^{2}, 0\right)$ such that $f_{t} \circ h=f_{t^{\prime}}$. This shows in particular that the bi-Lipschitz classification of real analytic function germs admits continuous moduli. The existence of such moduli for complex analytic function germs was shown by the authors in [2]. We recall that, on the other hand, the bi-Lipschitz equivalence of complex or real analytic set germs does not admit moduli by [6] and [7], [8].

In order to distinguish bi-Lipschitz types of complex analytic function germs of two complex variables $f:\left(\mathbb{C}^{2}, 0\right) \rightarrow(\mathbb{C}, 0)$ we construct in [2] a numerical invariant that is given in terms of the leading coefficients of the asymptotic expansions of $f$ along the branches of generic polar curve of $f$. This construction is recalled in Section 4 below. The

2000 Mathematics Subject Classification: 32S15, 32S05, $14 \mathrm{H} 15$.

The paper is in final form and no version of it will be published elsewhere. 
real case, though similar, is more delicate. Firstly we cannot simply use the invariants of the complexification. For instance the family $(0.1)$ for $t<0$ is bi-Lipschitz trivial but the family of complexification admits continuous moduli. This is due to the fact that polar curves $\partial f / \partial x=3\left(x^{2}-t y^{4}\right)=0, t<0$, that are responsible for the complex invariant, are invisible in the reals.

Given an analytic function germ $f:\left(\mathbb{R}^{n}, 0\right) \rightarrow(\mathbb{R}, 0)$, we associate to $f$ a family of germs of sets $(V(f), 0) \subset\left(\mathbb{R}^{n}, 0\right)$ defined by the condition that $|x||\operatorname{grad} f(x)|$ is comparable to the size of $|f(x)|$ on $V(f)$, see Sections 1 and 2 below. The sets $V(f)$ are preserved by bi-Lipschitz equivalence and give rise to a numerical invariant, see Section 3 and Theorem 5.1 below. This invariant is, in general, difficult to compute since it is not enough to use the branches of polar curve as in the complex case. Nevertheless, if $n=2$, then one may use the complexification to simplify the computation, see Proposition 5.3 below. We compute some examples in Section 6 and show, in particular, that in the family (0.1), $t>0$, our invariant changes continuously.

Notation and convention. We often write $r$ instead of $|x|$ which is the Euclidean norm of $x$. We use the standard notation $\varphi=o(\psi)$ or $\varphi=O(\psi)$ to compare the asymptotic behavior of $\varphi$ and $\psi$, usually when we approach 0 . We write $\varphi \sim \psi$ if $\varphi=O(\psi)$ and $\psi=O(\varphi)$, and $\varphi \simeq \psi$ if $\varphi / \psi$ tends to 1 . The gradient of a function $f$ will be denoted by $\nabla f$.

1. Characteristic exponents. Given an analytic function germ $f:\left(\mathbb{R}^{n}, 0\right) \rightarrow$ $(\mathbb{R}, 0)$, following [5] we associate to $f$ a finite set of positive rationals $L(f) \subset \mathbb{Q}^{+}$, called the characteristic exponents of $f$, that are defined as follows. The radial component of $\nabla f$ is defined on $\mathbb{R}^{n} \backslash\{0\}$ and equals

$$
\frac{\partial f}{\partial r} \frac{\partial}{\partial r}=\left(\sum_{i=1}^{n} \frac{x_{i}}{|x|} \frac{\partial f}{\partial x_{i}}\right) \sum_{i=1}^{n} \frac{x_{i}}{|x|} \frac{\partial}{\partial x_{i}}
$$

We shall often write $\partial_{r} f$ instead of $\partial f / \partial r$. The gradient $\nabla f$ splits into the orthogonal sum of its radial and spherical $\nabla^{\prime} f=\nabla f-(\partial f / \partial r)(\partial / \partial r)$ components.

Consider the germs at the origin of the sets

$$
W^{\varepsilon}:=\left\{x: f(x) \neq 0, \varepsilon\left|\nabla^{\prime} f\right| \leq\left|\partial_{r} f\right|\right\},
$$

where $\varepsilon>0$. Note that $W^{\varepsilon} \subset W^{\varepsilon^{\prime}}$ for $\varepsilon^{\prime}<\varepsilon$.

Let $\gamma:([0,1), 0) \rightarrow\left(\mathbb{R}^{n}, 0\right)$ be the germ of a real analytic curve. In order to simplify the notation we reparametrize $\gamma$ by the distance to the origin $\gamma(r)=\gamma(t(r))$ so that $|\gamma(t(r))|=r$. Then $\gamma(r)$ is a fractional power series (Puiseux expansion). We shall say for short that $\gamma(r)$ is an analytic arc at the origin. In the spherical coordinates we write

$$
\gamma(r)=r \theta(r)
$$

where $\theta(r)$ is a fractional power series, $|\theta(r)|=1$. Then the tangent vector to $\gamma(r)$ decomposes into the orthogonal sum of its radial and spherical components as follows

$$
\gamma^{\prime}(r)=\theta(r)+r \theta^{\prime}(r)
$$


and $r \theta^{\prime}(r)=o(1)$. We have a Puiseux expansion

$$
f(\gamma(r))=a_{\gamma} r^{l_{\gamma}}+\ldots
$$

$a_{l_{\gamma}} \neq 0, l_{\gamma} \in \mathbb{Q}^{+}$, and then by differentiating

$$
\frac{d f}{d r}(\gamma(r))=\partial_{r} f+\left\langle\nabla^{\prime} f, r \theta^{\prime}(r)\right\rangle \text {. }
$$

Suppose, moreover, that $\gamma(r) \in W^{\varepsilon}$ for $r>0$. Then $\partial_{r} f$ is dominant in the right-hand side term of (1.2) and hence

$$
\partial_{r} f=l_{\gamma} a_{\gamma} r^{l_{\gamma}-1}+o\left(r^{l_{\gamma}-1}\right) .
$$

Consequently, along any real analytic arc $\gamma$ in $W^{\varepsilon},\left(r \partial_{r} f / f\right)(x)$ tends to a positive rational $l_{\gamma}$. The set of all such limits being subanalytic and contained in $\mathbb{Q}^{+}$has to be finite and, see Section 4 of [5] for details, is independent of $\varepsilon$ for $\varepsilon>0$ and sufficiently small.

Proposition 1.1 ([5], Proposition 4.2). There exists a finite subset of positive rationals $L=\left\{l_{1}, \ldots, l_{k}\right\} \subset \mathbb{Q}^{+}$such that for any $\varepsilon>0$

$$
\frac{r \partial_{r} f}{f}(x) \rightarrow L \quad \text { as } \quad W^{\varepsilon} \ni x \rightarrow 0 .
$$

In particular, as a germ at the origin, each $W^{\varepsilon}$ is the disjoint union

$$
W^{\varepsilon}=\bigcup_{l_{i} \in L} W_{l_{i}}^{\varepsilon},
$$

where we may define $W_{l_{i}}^{\varepsilon}=\left\{x \in W^{\varepsilon}:\left|r \partial_{r} f / f-l_{i}\right| \leq r^{\delta}\right\}$, for $\delta>0$ sufficiently small.

Moreover, there exist constants $0<c_{\varepsilon}<C_{\varepsilon}$, which depend on $\varepsilon$, and such that

$$
c_{\varepsilon}<\frac{|f|}{r^{l_{i}}}<C_{\varepsilon} \quad \text { on } W_{l_{i}}^{\varepsilon} .
$$

The smallest characteristic exponent $\min _{l \in L} l$ equals the multiplicity $m_{f}$ of $f$ at the origin.

2. The sets $V$. Let $\delta>0$. Consider the germ at the origin of

$$
V^{\delta}:=\left\{x: f(x) \neq 0, \delta r|\nabla f| \leq|f| \leq \delta^{-1} r|\nabla f|\right\} .
$$

By Bochnak-Łojasiewicz Inequality [1] for any $0<c<m_{f}$, where $m_{f}$ denote the multiplicity of $f$ at the origin,

$$
c|f(x)| \leq r|\nabla f(x)|
$$

Hence if $0<\delta<m_{f}$ the second inequality of the definition of $V^{\delta}$ always holds. Let $L$ be the set of characteristic exponents of $f$ and let $l_{\max }=\max _{l \in L} l$.

Proposition 2.1.

(i) $W^{\varepsilon} \subset V^{\delta}$, if $\delta<\frac{1}{4}\left(l_{\max }\right)^{-1} \min \{1, \varepsilon\}, 0<\delta<m_{f}$.

(ii) $V^{\delta} \subset W^{\varepsilon}$, if $\varepsilon<\delta m_{f}$.

Proof. (i) On $W^{\varepsilon}$ we have $|f| \geq \frac{1}{2}\left(l_{\max }\right)^{-1} r\left|\partial_{r} f\right| \geq \frac{1}{2} \varepsilon\left(l_{\max }\right)^{-1} r\left|\nabla^{\prime} f\right|$ and consequently $|f| \geq \frac{1}{4}\left(l_{\max }\right)^{-1} \min \{1, \varepsilon\} r|\nabla f|$ as required. 
(ii) Let $\gamma(r)=r \theta(r)$ be an analytic arc, $\gamma(r) \in V^{\delta}$ for $r \neq 0$. By $r \theta^{\prime}(r)=o(1)$ we have always $\left\langle\nabla^{\prime} f, r \theta^{\prime}(r)\right\rangle=o(r|\nabla f(\gamma(r))|)$, and hence, since $f$ is of the same size as $r|\nabla f|$ on $\gamma,\left\langle\nabla^{\prime} f, r \theta^{\prime}(r)\right\rangle=o(f(\gamma(r)))$. Therefore, by (1.2), $r\left|\partial_{r} f\right| \simeq l_{\gamma}|f|$. Finally this implies

$$
r\left|\partial_{r} f\right| \simeq l_{\gamma}|f| \geq \delta l_{\gamma} r\left|\nabla^{\prime} f\right|
$$

and (ii) follows easily.

The equisingularity type of germs $\left(V^{\delta}, 0\right)$ does not depend on $\delta$ for $\delta>0$ and sufficiently small. In what follows we shall talk simply of the sets $V$, or $V(f)$, meaning $V^{\delta}$ for $\delta>0$ and small. Moreover, we may choose representatives of the germs by taking

$$
V^{\delta, r}:=\left\{x:|x|<r, x \in V^{\delta}\right\},
$$

where $0<r \ll \delta \ll 1$. For instance, by a connected component $\tilde{V}$ of $V$ we mean a connected component $\tilde{V}^{\delta, r}$ of $V^{\delta, r}$. One may show that for $r>0$ sufficiently small the connectivity of $\tilde{V}^{\delta, r}$ is equivalent to the connectivity of $\tilde{V}^{\delta, r} \cap S_{r^{\prime}}$, where $S_{r}$ denotes the sphere centered at the origin and of radius $0<r^{\prime}<r$.

Proposition 2.1 allows us to use the sets $V^{\delta}$ instead of $W^{\varepsilon}$ to define the characteristic exponents of $f$. In particular, by Propositions 1.1 and 2.1, each connected component of $V^{\delta}$ is contained in one of $W_{l}^{\varepsilon}$ and hence to each connected component of $V$ we may associate a unique characteristic exponent.

Given subanalytic $(X, 0) \subset\left(\mathbb{R}^{n}, 0\right)$, by the tangent cone of $X$ at the origin we mean

$$
C_{0}(X):=\overline{\left\{\left(t^{-1} x, t\right) \in \mathbb{R}^{n} \times \mathbb{R}: t>0, x \in X\right\}} \cap\left(\mathbb{R}^{n} \times\{0\}\right)
$$

that we consider as a subset of $\mathbb{R}^{n}$. It is a semi-cone that is it is stable by multiplication by positive reals. By the Eojasiewicz Inequality, as the germ at the origin, $X$ is contained in a cuspidal neighborhood of $C_{0}(X)$, that is, there is $\eta>0$ such that

$$
X \subset\left\{x \in \mathbb{R}^{n}: \operatorname{dist}\left(x, C_{0}(X)\right)<|x|^{1+\eta}\right\} .
$$

Let $V^{\prime}$ be a connected component of $V$ such that the tangent cone $C_{0}\left(V^{\prime}\right)$ is a half-line. Suppose that $V^{\prime}$ is L-regular in the sense of [7]. Then there is $M>0$ such that for any $x, x^{\prime} \in V^{\prime}$ there is a continuous subanalytic curve $\xi(t)$ connecting $x$ and $x^{\prime}$ in $V^{\prime}$ and of length less than or equal to $M\left|x-x^{\prime}\right|$. Suppose $|x|=\left|x^{\prime}\right|=r$. Then, by (2.2) for $X=V^{\prime}$, $|\xi(t)-x| \leq M\left|x-x^{\prime}\right| \leq M^{\prime} r^{1+\eta}$ and $|\xi(t)| \geq r-M^{\prime} r^{1+\eta} \geq \frac{1}{2} r$ for $r$ small. By the definition of $V^{\delta}$,

$$
\left|\frac{d}{d t} f(\xi(t))\right|=\left|\left\langle\nabla f, \xi^{\prime}(t)\right\rangle\right| \leq|\nabla f|\left|\xi^{\prime}(t)\right| \leq \delta^{-1} \frac{|f(\xi(t))|\left|\xi^{\prime}(t)\right|}{|\xi(t)|} .
$$

Hence

$$
\left|\frac{d}{d t} \ln \right| f(\xi(t))|| \leq \frac{2 \delta^{-1}}{r}\left|\xi^{\prime}(t)\right| .
$$

By integration, $|\ln | f(x)|-\ln | f\left(x^{\prime}\right)|| \leq 2 \delta^{-1} r^{-1} \operatorname{length}(\xi) \leq 2 \delta^{-1} M r^{\eta}$, which gives

$$
\left|\frac{f(x)}{f\left(x^{\prime}\right)}\right| \leq e^{\tilde{M} r^{\eta}} .
$$

If $V^{\prime}$ is not L-regular it is a union of finitely many L-regular sets [7], [4], [8] and this can be used to show (2.3) for any connected $V^{\prime}$. Hence, by symmetry and the fact that the 
sign of $f$ is constant on $V^{\prime}$, for small $r$

$$
1-C r^{\eta} \leq \frac{f(x)}{f\left(x^{\prime}\right)} \leq 1+C r^{\eta} .
$$

This shows the following.

Proposition 2.2. Let $V^{\prime}$ be a connected component of $V$ such that $C_{0}\left(V^{\prime}\right)$ is a halfline. Let $l=l_{V^{\prime}}$ be the characteristic component associated to $V^{\prime}$. Then there is $a \neq 0$ such that

$$
f(x)=a r^{l}+O\left(r^{l+\delta}\right)
$$

for all $x \in V^{\prime}$ and an exponent $\delta>0$.

We shall call the constant $a$ the leading coefficient associated to $V^{\prime}$ and the term ar ${ }^{l}$ the leading term of $f$ on $V^{\prime}$.

3. Invariants of bi-Lipschitz equivalence. Let $f, g:\left(\mathbb{R}^{n}, 0\right) \rightarrow(\mathbb{R}, 0)$ be two analytic function germs. Let $h:\left(\mathbb{R}^{n}, 0\right) \rightarrow\left(\mathbb{R}^{n}, 0\right)$ be the germ of a bi-Lipschitz homeomorphism such that $f=g \circ h$. Fix $L \geq 1$, a common Lipschitz constant of $h$ and $h^{-1}$. Then

$$
L^{-1}|\nabla g(h(x))| \leq|\nabla f(x)| \leq L|\nabla g(h(x))| .
$$

This shows that for $\delta>0$

$$
V^{L^{2} \delta}(g) \subset h\left(V^{\delta}(f)\right) \subset V^{L^{-2} \delta}(g) .
$$

We shortly say that the sets $V$ are preserved by bi-Lipschitz equivalence. The sets $V$ provide a numerical invariant of bi-Lipschitz equivalence that changes continuously in some analytic families of analytic function germs. We shall construct this invariant below. The property (3.1) shows, in particular, that $h$ gives a one-to-one correspondence between the connected components of $V(f)$ and $V(g)$ and it is easy to see that the associated characteristic exponents are preserved. In what follows we restrict ourselves to the components whose tangent cones are half-lines. Note that this property is also preserved by bi-Lipschitz homeomorphisms.

Suppose that two such connected components $V_{1}(f), V_{2}(f)$ of $V(f)$ are tangent, i.e. their tangent cones at the origin coincide, and that their characteristic exponents are equal to $l \in \mathbb{Q}^{+}$. By Proposition 2.2

$$
f(x)=a_{i} r^{l}+o\left(r^{l}\right) \quad \text { for } x \in V_{i}(f),
$$

$i=1,2$. Denote by $V_{1}(g), V_{2}(g)$ the corresponding connected components of $V(g)$. Then

$$
f(x)=g(h(x))=b_{i}|h(x)|^{l}+o\left(|h(x)|^{l}\right) \quad \text { for } x \in V_{i}(f),
$$

where $b_{i}$ is the leading coefficient of the expansion of $g\left(x_{i}\right)$ on $V_{i}(g)$. For $i=1,2$, choose an analytic arc $x_{i}(r)$ so that $x_{i}(r) \in V_{i}(f)$ for $r>0$. By tangency of $V_{1}(f)$ and $V_{2}(f)$ there is $\eta>0$ such that

$$
\left|x_{1}(r)-x_{2}(r)\right|=O\left(r^{1+\eta}\right) .
$$

Similarly, since $V_{1}(g), V_{2}(g)$ have to be tangent,

$$
\left|h\left(x_{1}(r)\right)-h\left(x_{2}(r)\right)\right|=O\left(\left|h\left(x_{1}(r)\right)\right|^{1+\eta}\right)=O\left(r^{1+\eta}\right) .
$$


Thus the limit

has to be equal to the limit

$$
\frac{f\left(x_{1}(r)\right)}{f\left(x_{2}(r)\right)} \rightarrow \frac{a_{1}}{a_{2}} \quad \text { as } r \rightarrow 0
$$

$$
\frac{g\left(h\left(x_{1}(r)\right)\right)}{g\left(h\left(x_{2}(r)\right)\right)} \rightarrow \frac{b_{1}}{b_{2}} \quad \text { as } r \rightarrow 0 .
$$

Corollary 3.1. The quotient of leading coefficients associated to $V_{1}(f)$ and $V_{2}(f)$ is a bi-Lipschitz invariant.

REMARK 3.2. The leading coefficients themselves are not bi-Lipschitz invariants. They may be changed, for instance, by a linear map $h(x)=A x$.

4. Complex case. We recall the main result of $[2]$. Let $f(x, y):\left(\mathbb{C}^{2}, 0\right) \rightarrow(\mathbb{C}, 0)$ be a germ of analytic function with Taylor expansion:

$$
f(x, y)=H_{k}(x, y)+H_{k+1}(x, y)+\ldots,
$$

$k$ being the multiplicity of $f$ at the origin. We shall assume $f(x, y)$ is mini-regular in $x$ of order $k$, that is, that $H_{k}(1,0) \neq 0$. We also assume, for simplicity, that $f(x, y)$ has no multiple roots.

By a (complex) analytic arc we mean a fractional power a series of the form

$$
\lambda: x=\lambda(y):=c_{1} y^{n_{1} / N}+c_{2} y^{n_{2} / N}+\ldots, \quad c_{i} \in \mathbb{C},
$$

where $N \leq n_{1}<n_{2}<\ldots$ are positive integers having no common divisor, such that $\lambda\left(t^{N}\right)$ has positive radius of convergence. We can identify $\lambda$ with the analytic arc $\lambda: x=$ $c_{1} t^{n_{1}}+c_{2} t^{n_{2}}+\ldots, y=t^{N},|t|$ small, which is not tangent to the $x$-axis (since $n_{1} / N \geq 1$ ).

A polar arc $x=\gamma(y)$ is a branch of the polar curve $\Gamma: \partial f / \partial x=0$. Since $f$ is mini-regular in $x, x=\gamma(y)$ is not tangent to the $x$-axis and it is an arc in our sense. Let $\gamma$ be a polar arc. We associate to $\gamma$ two numbers: $l=l(\gamma) \in \mathbb{Q}_{+}$and $a=a(\gamma) \in \mathbb{C}^{*}$ given by the expansion

$$
f(\gamma(y), y)=a y^{l}+\ldots
$$

If $l>k$ then the polar arc has to be tangent to the singular locus of the tangent cone $C_{0}(X), X=f^{-1}(0)$, given by $\operatorname{Sing}\left(C_{0}(X)\right):=\left\{\partial H_{k} / \partial x=\partial H_{k} / \partial y=0\right\}$. We call such polar arcs tangential.

Fix a line $\Lambda \subset \operatorname{Sing}\left(C_{0}(X)\right)$. Let $\Gamma(\Lambda)$ denote the set of polar arcs tangent to $\Lambda$. Associate to $\Lambda$ the set of formal expressions

$$
\mathcal{I}(\Lambda)=\left\{a(\gamma) y^{l(\gamma)}: \gamma \in \Gamma(\Lambda)\right\} / \mathbb{C}^{*}
$$

where $\mathbb{C}^{*}$ acts by multiplication on $y$ :

$$
\left\{a_{1} y^{l_{1}}, \ldots, a_{m} y^{l_{m}}\right\} \sim\left\{\left(a_{1} c^{l_{1}}\right) y^{l_{1}}, \ldots,\left(a_{m} c^{l_{m}}\right) y^{l_{m}}\right\}
$$

By the invariant $\operatorname{Inv}(f)$ of $f$ we mean the set of all $\mathcal{I}(\Lambda)$, where $\Lambda$ runs over all lines in $\operatorname{Sing}\left(C_{0}(X)\right) \cdot \operatorname{Inv}(f)$ is well-defined and does not depend on the choice of local coordinates. The main result of [2] is the following.

TheOrem 4.1. Let $f_{1}, f_{2}$ be two functions germs $\left(\mathbb{C}^{2}, 0\right) \rightarrow(\mathbb{C}, 0)$. If $f_{1}$ and $f_{2}$ are bi-Lipschitz equivalent then $\operatorname{Inv}\left(f_{1}\right)=\operatorname{Inv}\left(f_{2}\right)$. 
5. From complex to real. Let $f(x, y):\left(\mathbb{R}^{2}, 0\right) \rightarrow(\mathbb{R}, 0)$ be an analytic function germ. Similarly to the complex case we suppose that $f$ is mini-regular in $x$, that is, $H_{k}(1,0) \neq 0$ for $H_{k}$ given by the Taylor expansion (4.1) of $f$ at the origin and that the complexification of $f$ has no multiple roots.

Consider a connected component $V^{\prime}$ of $V(f)$ and suppose that the associated characteristic exponent $l=l\left(V^{\prime}\right)>k$. Then $V^{\prime}$ is tangent to $C_{0}(X)=\left\{H_{k}(x, y)=0\right\}$, $X=f^{-1}(0)$, that is, $C_{0}\left(V^{\prime}\right)$ is a half-line contained in $C_{0}(X)$. Indeed, if $\gamma(r)$ is an analytic arc at the origin not tangent to $C_{0}(X)$ then

$$
|f(\gamma(r))| \sim r^{k}, \quad|\nabla f(\gamma(r))| \sim r^{k-1} .
$$

If $\gamma(r)$ is tangent to $C_{0}(X)$ but not tangent to $\operatorname{Sing}\left(C_{0}(X)\right)$ then

$$
|f(\gamma(r))|=o\left(r^{k}\right), \quad|\nabla f(\gamma(r))| \sim r^{k-1} .
$$

and hence $\gamma$ does not intersect $V$. That shows that $C_{0}\left(V^{\prime}\right)$ has to be contained in $\operatorname{Sing}\left(C_{0}(X)\right)$.

Fix a half-line $\lambda \subset \operatorname{Sing}\left(C_{0}(X)\right)$. Let $\mathcal{V}(\lambda)$ denote the set of connected components of $V$ tangent to $\lambda$. For such a component $V^{\prime}$ we denote by $l\left(V^{\prime}\right)$, resp. $a\left(V^{\prime}\right)$, the characteristic exponent, resp. the leading coefficient, associated to $V^{\prime}$. We associate to $\lambda$ the set of formal expressions

$$
\mathcal{I}(\lambda)=\left\{a\left(V^{\prime}\right) r^{l\left(V^{\prime}\right)}: V^{\prime} \in \mathcal{V}(\lambda)\right\} / \mathbb{R}_{>0},
$$

where $c \in \mathbb{R}_{>0}$ acts by multiplication on $r$ as in (4.4). By the invariant $\operatorname{Inv}(f)$ of $f$ we mean the set of all $\mathcal{I}(\lambda)$, where $\lambda$ runs over all half-lines in $\operatorname{Sing}\left(C_{0}(X)\right)$. The argument at the end of Section 3 above, see also [2], Section 4, gives the following analog of Theorem 4.1.

TheOREM 5.1. Let $f_{1}, f_{2}$ be two function germs $\left(\mathbb{R}^{2}, 0\right) \rightarrow(\mathbb{R}, 0)$. If $f_{1}$ and $f_{2}$ are bi-Lipschitz equivalent then $\operatorname{Inv}\left(f_{1}\right)=\operatorname{Inv}\left(f_{2}\right)$.

Let $\gamma: x=\gamma(y)$ be a real polar arc, that is, a half-branch of the real polar curve $\Gamma(f): \partial f / \partial x=0$ given by a Puiseux expansion $x=\gamma(y), y \geq 0$. Its tangent half-line at the origin is spanned by $\left(\gamma^{\prime}(0), 1\right)$. Write

$$
f(\gamma(y), y)=a y^{l}+o\left(y^{l}\right)
$$

Then, along $\gamma$,

$$
a l y^{l-1}+\ldots=\frac{d f}{d y}=\gamma^{\prime}(y) \partial f / \partial x+\partial f / \partial y=\partial f / \partial y .
$$

Since $r \simeq\left|\left(\gamma^{\prime}(0), 1\right)\right||y|$ on $\gamma$, this shows that $(\gamma(y), y) \in V$ for $y>0$. Thus we obtain the following.

\section{Proposition 5.2. $\Gamma(f) \backslash\{0\} \subset V$.}

Moreover, the exponent $l$ of (5.2) is the characteristic exponent associated to the connected component $V^{\prime}$ of $V$ that contains $\gamma$. If $l>k$ then the leading coefficient associated to $V^{\prime}$ equals $a\left|\left(\gamma^{\prime}(0), 1\right)\right|^{-l}$. This allows us to recover partly $\operatorname{Inv}(f)$ using the half-branches of the polar curve. But unlike as in the complex case it may happen that some part of invariant cannot be seen on the real polar curve. Then we may use the 
complexification of $f$. Note that one may similarly define the set $V$ for complex analytic functions. It is a bi-Lipschitz invariant by the same argument. And, using the Newton algorithm as in [2], Section 5, one may show that any connected component of $V$ contains a complex polar arc.

Let $\Lambda$ be the complexification of a real line in $\operatorname{Sing}\left(C_{0}(X)\right)$. The real part of $\Lambda$ contains two half-lines that we may distinguish using the given system of coordinates: $\lambda_{+}$is the one that is the graph of a linear function over $y \geq 0$ and $\lambda_{-}$is the graph of the same function over $y \leq 0$. Then the invariants $\mathcal{I}\left(\lambda_{ \pm}\right)$of $f$ and the leading terms of the complexification along the polar arcs in $\Gamma(\Lambda)$ are related by the following result.

Proposition 5.3.

$$
\mathcal{I}\left(\lambda_{ \pm}\right) \subset\left\{a(\gamma) y^{l(\gamma)}: \gamma \in \Gamma(\Lambda)\right\} / \mathbb{R}_{>0},
$$

where we identify $r$ with $\pm y$.

Proof. We show the formula for $\lambda_{+}$equal to the positive $y$-axis. Choose an analytic arc $\xi$ in $V \cup\{0\}$ tangent to the $y$-axis. Then the complexification of $\xi$ is an analytic arc that we denote also by $\xi: x=\xi(y)$. Let $f_{\mathbb{C}}$ denote the complexification of $f$. Since $V(f)$ is contained in $V\left(f_{\mathbb{C}}\right)$ so is $(\xi(y), y)$, for $y \neq 0$. By assumptions, $\operatorname{ord}_{0} \xi(y)>1$, $\operatorname{ord}_{0} f(\xi(y), y)>k$. Then there is a complex polar arc tangent to the $y$-axis and with the same leading term of the asymptotic expansion of $f$, see the first case of proof of Theorem 5.3 of [2]. This shows the proposition.

\section{Examples.}

Example 6.1 (Compare [2], Section 3). Let $f_{t}(x, y)=x^{3}-3 t x y^{4}+y^{6}, t>0$, $1-4 t^{3} \neq 0$. The polar curve $\partial f / \partial x=3\left(x^{2}-t y^{4}\right)=0$ has two analytic branches $x= \pm \sqrt{t} y^{2}$ and

$$
f_{t}\left( \pm \sqrt{t} y^{2}, y\right)=\left(1 \mp 2 t^{3 / 2}\right) y^{6}
$$

We have two characteristic exponents $l=3$ and $l=6$. For $\lambda$ being the positive or negative $y$-axis the invariant equals

$$
\mathcal{I}(\lambda)=\left\{1+2 t^{3 / 2}, 1-2 t^{3 / 2}\right\} / \mathbb{R}_{>0} .
$$

Thus if $f_{t}$ and $f_{t^{\prime}}$ are bi-Lipschitz equivalent then $\left(1+2 t^{3 / 2}\right) /\left(1-2 t^{3 / 2}\right)$ is equal to $\left(1+2 t^{\prime 3 / 2}\right) /\left(1-2 t^{\prime 3 / 2}\right)$ or $\left(1-2 t^{\prime 3 / 2}\right) /\left(1+2 t^{\prime 3 / 2}\right)$. The latter is impossible for $t, t^{\prime}>0$ and the former implies $t=t^{\prime}$.

ExAmple 6.2. Let $f_{t}(x, y)=x^{3}+3 t x y^{4}+y^{6}, t>0$. The polar curve $\partial f / \partial x=$ $3\left(x^{2}+t y^{4}\right)=0$ has no real branch. There is only one characteristic exponent $l=3$. Indeed, another possibility allowed by the complexification of $f$ is $l=6$. But $|\nabla f| \geq$ $\partial f / \partial x=3\left(x^{2}+t y^{4}\right)$ is much bigger than $r^{6-1}$.

Actually one may show that $f_{t}$ is Lipschitz trivial. It can be trivialized by the Kuo vector field

$$
\vec{v}(x, y, t)=\partial / \partial t-\frac{\partial f / \partial t}{\left|\nabla f_{t}\right|^{2}} \nabla f_{t}
$$

where $\nabla f_{t}$ denotes the gradient only in $x, y$ variables. Then $\vec{v}$ is Lipschitz and its flow gives a bi-Lipschitz trivialization of $f_{t}$. 
ExAmPLE 6.3. The functions $f_{t}(x, y)$ of Example 6.1 are Newton nondegenerate and the family $f_{t}$ admits a simultaneous toric resolution. Consider $f(x, y)=x^{3}+y^{6}$, $g(x, y)=x^{3}-y^{6}$. These two functions can also be embedded in a family that can be simultaneously resolved by a toric resolution, see [3], and hence they are blow-analytically equivalent. But they are not bi-Lipschitz equivalent. Indeed, in both cases the polar curves $\partial f / \partial x=\partial g / \partial x=3 x^{2}=0$ reduces to the $y$-axis and $f(0, y)=y^{6}, g(0, y)=-y^{6}$. For the characteristic exponent $l=6$, by Proposition 5.3, there is only one leading coefficient, that is, positive for $f$ and negative for $g$. Thus, by Theorem 5.1, $f$ is not bi-Lipschitz equivalent to $g$.

\section{References}

[1] J. Bochnak, S. Łojasiewicz, A converse of the Kuiper-Kuo theorem, in: Proceedings of Liverpool Singularities Symposium, I (1969/70), Lecture Notes in Math. 192, Springer, Berlin, 1971, 254-261.

[2] J.-P. Henry, A. Parusiński, Existence of moduli for bi-Lipschitz equivalence of analytic functions, Compositio Math. 136 (2003), 217-235.

[3] S. Koike, A. Parusiński, Motivic-type invariants of blow-analytic equivalence, Ann. Inst. Fourier (Grenoble) 53 (2003), 2061-2104.

[4] K. Kurdyka, On a subanalytic stratification satisfying a Whitney property with exponent 1 , in: Real Algebraic Geometry (Rennes, 1991), Lecture Notes in Math. 1524, Springer, Berlin, 1992, 316-322.

[5] K. Kurdyka, T. Mostowski, A. Parusiński, Proof of the Gradient Conjecture of R. Thom, Ann. of Math. (2) 52 (2000), 763-792.

[6] T. Mostowski, Lipschitz Equisingularity, Dissertationes Math. (Rozprawy Mat.) 243, PWN, Warszawa, 1985.

[7] A. Parusiński, Lipschitz properties of semi-analytic sets, Ann. Inst. Fourier (Grenoble) 38 (1988), 189-213.

[8] A. Parusiński, Lipschitz stratifications of subanalytic sets, Ann. Sci. École Norm. Sup. (4) 27 (1994), 661-696. 